OPEN ACCESS

Edited by:

Syed Khizer Hasan, Advanced Centre for Treatment, Research and Education in Cancer.

Reviewed by:

Shilpee Dutt,

Advanced Centre for Treatment, Research and Education in Cancer,

India

Sanjeev Kuumar Gupta, All India Institute of Medical Sciences,

India

Papagudi Subramanian, Advanced Centre for Treatment, Research and Education in Cancer,

India

${ }^{*}$ Correspondence: Yordan Sbirkov

yordan.sbirkov@mu-plovdiv.bg Victoria Sarafian

victoria.sarafian@mu-plovdiv.bg

Specialty section:

This article was submitted to Hematologic Malignancies,

a section of the journal

Frontiers in Oncology

Received: 22 November 2020 Accepted: 02 February 2021

Published: 15 March 2021

Citation:

Sbirkov $Y$, Ivanova T, Burnusuzov $H$, Gercheva K, Petrie K, Schenk T and

Sarafian V (2021) The Protozoan Inhibitor Atovaquone Affects Mitochondrial Respiration and Shows In Vitro Efficacy Against

Glucocorticoid-Resistant Cells in

Childhood B-Cell Acute Lymphoblastic Leukaemia.

Front. Oncol. 11:632181.

doi: 10.3389/fonc.2021.632181

\section{The Protozoan Inhibitor Atovaquone Affects Mitochondrial Respiration and Shows In Vitro Efficacy Against Glucocorticoid-Resistant Cells in Childhood B-Cell Acute Lymphoblastic Leukaemia}

\author{
Yordan Sbirkov ${ }^{1,2 *}$, Tsvetomira Ivanova ${ }^{2}$, Hasan Burnusuzov ${ }^{2,3,4}$, Kalina Gercheva ${ }^{1}$, \\ Kevin Petrie ${ }^{5}$, Tino Schenk ${ }^{6,7}$ and Victoria Sarafian ${ }^{1,2 *}$ \\ ${ }^{1}$ Department of Medical Biology, Medical University of Plovdiv, Plovdiv, Bulgaria, ${ }^{2}$ Research Institute at Medical University of \\ Plovdiv, Medical University of Plovdiv, Plovdiv, Bulgaria, ${ }^{3}$ Department of Pediatrics and Medical Genetics, Medical University \\ of Plovdiv, Plovdiv, Bulgaria, ${ }^{4}$ Center for Competence Personalized Innovative Medicine (PERIMED), Medical University of \\ Plovdiv, Plovdiv, Bulgaria, ${ }^{5}$ Faculty of Health Sciences and Wellbeing, School of Medicine, University of Sunderland, \\ Sunderland, United Kingdom, 6 Department of Hematology and Medical Oncology, Jena University Hospital, Jena, Germany, \\ 7 Institute of Molecular Cell Biology, Center for Molecular Biomedicine Jena (CMB), Jena University Hospital, Jena, Germany
}

Childhood acute lymphoblastic leukaemia (CALL) accounts for about one third of all paediatric malignancies making it the most common cancer in children. Alterations in tumour cell metabolism were first described nearly a century ago and have been acknowledged as one of the key characteristics of cancers including CALL. Two of the backbone chemotherapeutic agents in the treatment of this disease, Glucocorticoids and L-asparaginase, are exerting their anti-leukaemic effects through targeting cell metabolism. Even though risk stratification and treatment regimens have improved cure rates to nearly $90 \%$, prognosis for relapsed children remains poor. Therefore, new therapeutic approaches are urgently required. Atovaquone is a well-tolerated drug used in the clinic mainly against malaria. Being a ubiquinone analogue, this drug inhibits co-enzyme Q10 of the electron transport chain (ETC) affecting oxidative phosphorylation and cell metabolism. In this study we tested the effect of Atovaquone on CALL cells in vitro. Pharmacologically relevant concentrations of the inhibitor could effectively target mitochondrial respiration in both cALL cell lines (REH and Sup-B15) and primary patient samples. We found that Atovaquone leads to a marked decrease in basal respiration and ATP levels, as well as reduced proliferation, cell cycle arrest, and induction of apoptosis. Importantly, we observed an enhanced anti-leukaemic effect when Atovaquone was combined with the standard chemotherapeutic Idarubicin, or with Prednisolone in an in vitro model of Glucocorticoid resistance. Repurposing of this clinically approved inhibitor renders further investigations, but also presents opportunities for fast-track trials as a single agent or in combination with standard chemotherapeutics.

Keywords: metabolism, mitochondria, acute B-cell lymphoblastic leukaemia, glucocorticoid resistance, atovaquone 


\section{INTRODUCTION}

Altered cancer cell metabolism, described nearly a century ago by Warburg (1), has gone a long way from an intriguing scientific observation to a validated drug target in the clinic. Initially believed to serve as an alternative energy source for the rapidly dividing cancer cells, "Warburg's effect" of oxidative glycolysis is now considered an effective means of metabolic rewiring which provides multiple precursors for key biosynthetic pathways (2-4). Excellent examples showing alterations in cell bioenergetics and the clinical use of certain metabolic vulnerabilities come from acute lymphoblastic leukaemia (ALL) (4).

Childhood ALL (cALL) accounts for about one third of all paediatric cancers. The disease is characterised by undifferentiated highly proliferative lymphoid cells of B-cell ( $~ 85 \%)$, T-cell (10-15\%) or mixed lineage $(<5 \%)$. The genetic and epigenetic aberrations underlying this malignancy are marked by great heterogeneity among patients with a recent study highlighting that there may be over 20 sub-types of B-cell cALL (5). Furthermore, there are a number of metabolic alterations in cALL present already at diagnosis. Some examples include aberrant transcriptional profile involving upregulation of glycolysis and downregulation of citric acid cycle genes (6), mutations in mitochondrial DNA affecting Oxidative Phorphorylation (OxPhos) (7), activation of the AKT pathway in T-ALL, which can cause upregulation of glycolysis and metabolic rewiring $(8,9)$, and others. Many of the standard chemotherapeutics can target cell metabolism. Glucocorticoids (GCs), the backbone of induction therapy, are effectively decreasing glucose uptake and shifting metabolism towards glutamine synthesis and fatty acid oxidation (10, 11). Anthracyclins inhibit topoisomerase II, but can also block Complex I of the electron transport chain (ETC) decreasing mitochondrial function $(11,12)$. L-Asparaginase is another critical part of standard treatment. It depletes extracellular asparagine levels, but also leads to glutamine deamination (13), glycolysis, and pyrimidine synthesis (14). Lastly, Methotrexate inhibits the folic acid cycle, thus the synthesis of nucleotides (15).

Refined risk group stratification and treatment regimens, including targeting of metabolic vulnerabilities in blast cells, have improved overall survival to $85-90 \%$ (16). Nevertheless, 5year event-free survival after relapse for certain sub-groups of patients (e.g. with isolated bone marrow relapse, or deletion of IKZF1) may be less than 30\% (17). Therefore, novel therapeutic approaches are urgently required especially for high risk groups, refractory disease, and relapsed patients.

Drug repurposing is a strategy whereby a drug, which is already approved for the treatment of a certain condition, is transferred into a different disease context. The advantage of this approach is saving considerable amount of time (at least 2-3 times quicker (18)) and money (3-10 times less on average (19)), ultimately translating into saving more lives faster and at a lower cost. Encouraging examples of drug repositioning already in use or in clinical trials range from Aspirin in colorectal cancer (20), Metformin (for diabetes) and Daunorubicin (antibiotic) for various malignancies $(20,21)$, retinoic acid (vitamin A) in acute promyelocytic leukaemia (APL) (22), a combination of retinoic acid and Tranylcypromine (TCP-anti-depressant) in acute myeloid leukaemia (23), and others.

Atovaquone (Ato) is an analogue of ubiquinone (the oxidised form of coenzyme Q10) making it an effective inhibitor of complex III of the electron transport chain (ETC) (24). Due to its high specificity and excellent tolerability Ato has been used against protozoa like Plasmodium spp and Toxoplasma gondii, turning it into a key component of standard anti-malarial therapy including in children (25). Interestingly, in recent years Ato has been highlighted as a potent anti-proliferative and apoptotic agent in different malignancies. For instance, Ato has proved effective in vitro in breast cancer (26), in pharyngeal and colorectal cancer cell lines in enhancing the effect of radiotherapy (27), in retinoblastoma cells (28), and in haematologic malignancies like chronic lymphoblastic leukaemia (29) and acute myeloid leukaemia (30). Nevertheless, the effect of Ato on CALL has not been studied.

Therefore, given the body of evidence in literature that cALL cells have alterations in their mitochondrial and metabolic function, together with encouraging results using Ato in other malignancies, the aim of our study was to investigate if Ato may be effective against cALL cells as well.

\section{MATERIALS AND METHODS}

\section{Cell Culture, Drug Treatments, Cell Viability, and Cell Counts}

The childhood ALL cell line REH (harbouring a TEL-AML1 fusion, isolated after $1^{\text {st }}$ relapse) was kindly provided by Dr. Tino Schenk from the University Hospital of Jena (Germany), whilst Sup-B15 was purchased from DSMZ (Germany). Both cell lines were cultured in standard conditions-REH cells in RPMI with $10 \%$ foetal bovine serum (FBS) and $0.5 \%$ PenicillinStreptomycin (Pen/Strep), and Sup-B15 (harbouring a BCRABL1 fusion, isolated after $2^{\text {nd }}$ relapse) in IMDM with $20 \%$ FBS and $0.5 \%$ Pen/Strep (all from PAN-Biotech, Germany). Atovaquone, Idarubicin, and Prednisolone (Cayman Chemical, USA, European Division in Estonia) were dissolved in DMSO (PAN-Biotech, Germany) to 20,10 , and $10 \mu \mathrm{M}$ respectively. $\mathrm{IC}_{50}$ values were determined following drug treatment of 3 days and assessment of cell viability with MTT assay. All experiments were performed at least in biological duplicates and technical triplicates. Cell counts were determined using Luna II cell counter (Logosbio, South Korea).

\section{Establishment of Stable Prednisolone- Resistant Cells}

To develop Prednisolone-resistant sub-clones of Sup-B15 cells, we used alternating cycles of drug treatment with increasing concentrations of Prednisolone by small increments followed by cell expansion. In brief, the $\mathrm{IC}_{50}$ concentration of Prednisolone for Sup-B15 was determined, and cells were treated with this concentration of $0.002 \mu \mathrm{M}$ for 3 days. Surviving cells were centrifuged to eliminate dead cells, cell debris, and any remaining Prednisolone, washed with medium, and left to recover and expand in growth medium for 3-4 days. These 
cells were then treated with $1.5 \times \mathrm{IC}_{50}$ of Prednisolone for another 3 days, then centrifuged, washed, and left in growth medium to expand again. The third cycle used $2 \times \mathrm{IC}_{50}$ of Prednisolone, and every following cycle used increments of $30-50 \%$ higher concentration, compared to the previous cycle. Every four to five cycles Prednisolone was titrated to determine the shift in $\mathrm{IC}_{50}$ compared to the parental Sup-B15 cells. These cycles of treatment with increased concentrations of Prednisolone followed by cell recovery and expansion (>15 cycles) were repeated until we reached about 1,000 times resistance compared to parental Sup-B15 cells.

\section{Patient Samples}

Bone marrow aspirates from patients were collected for standard diagnosis by flow cytometry. Cells from the remaining samples were isolated using Pancoll (PAN-Biotech) following the instructions by the manufacturer. At least 300,000 cells per well were plated for Atovaquone treatment, cultured for 3 days and analysed with the Seahorse XFp Instrument. Where possible, 30,60 , and $90 \mu \mathrm{M}$ of Atovaquone were tested. For assessment of viability and the effect of Atovaquone and Idarubicin combinations, patient samples were seeded at $\sim 50,000$ cells per well in 96-well plates. All experiments with patient samples were performed in technical duplicates and triplicates where cell numbers allowed. Samples were taken after informed consent by the parents/guardians of the patients, and the study was approved by the Ethics Committee at the Medical University of Plovdiv.

\section{Seahorse XFp Analysis of Mitochondrial Function}

For assessment of mitochondrial function we used Seahorse XFp analyser and Mito Stress Test kit (Agilent, USA). For this Mito Stress protocol, three mitochondrial inhibitors (all from Cayman Chemical, USA, European Division in Estonia) were injected consecutively onto the cells-Oligomycin (inhibitor of the ATP Synthetase) at $1 \mu \mathrm{M}$ final concentration, FCCP (trifluoromethoxy carbonylcyanide phenylhydrazone-an uncoupler of the ETC) at $2 \mu \mathrm{M}$ final concentration and Rotenone (inhibitor of Complex I of the ETC) at $0.5 \mu \mathrm{M}$ final concentration. This allowed the assessment of several parameters including basal and maximal respiration, spare respiratory capacity, ATP levels, and others. Bcell ALL cells were grown in 12- or 24-well plates with or without Atovaquone for $72 \mathrm{~h}$. Cells were then centrifuged and washed twice with Seahorse-compatible bicarbonate buffer-free RPMI (AppliChem, Germany) supplemented with Pyruvate (1 mM) with adjusted final $\mathrm{pH}$ of 7.2-7.4. Cells were then counted with Luna II cell counter (LogosBio, South Korea) and seeded at 100300,000/well in Seahorse microplates (Agilent, USA), which were pre-coated with Poly-D-Lysine at $1 \mathrm{mg} / \mathrm{ml}$ for $30 \mathrm{~min}$. Seahorse oxygen consumption rate (OCR) data for each experiment was normalised to total live cells per well counted with Luna II cell counter (LogosBio, South Korea) using Seahorse Wave Desktop Software v 2.6.0 (Agilent, USA). Data analysis was then performed using the XF Mito Stress Report Generator within Wave Desktop Software.

\section{Cell Cycle and Apoptosis Assays}

Cell cycle analysis and Annexin V/PI assay for apoptosis were carried out on a Guava ${ }^{\circledR}$ Muse ${ }^{\circledR}$ Cell Analyser (Luminex, USA) using the respective standard kits and protocols optimised by Luminex.

\section{RNA-Sequencing}

RNA extraction from control untreated and Ato-treated $(30 \mu \mathrm{M})$ samples in biological triplicates was carried out on day 3 after treatment from $\sim 1$ million pelleted cells per condition with Qiagen RNeasy kit (Qiagen, USA) according to the manufacturer's recommendations. Initial sample concentration and quality was assessed by Nanodrop (Thermo, USA). Samples were then sent to Novogene UK for further QC, reverse transcription, library preparation, and sequencing. In brief, NEBNext ${ }^{\circledR}$ UltraTM RNA Library Prep Kit for Illumina ${ }^{\circledR}$ (NEB, USA) was used for library preparation with AMPure XP beads (Beckman Coulter, Beverly, USA) for size selection and PCR purification steps, both performed as instructed by the manufacturers. Finally, sequencing was performed on an Illumina Sequencer generating $>20 \mathrm{M}$ pair-end clean reads per sample.

\section{Synergy Testing}

Analysis of drug combinations was carried out with SynergyFinder (31). The zero interaction potency (ZIP) model (32) was chosen as the default parameter in SynergyFinder (together with four parameter logistic regression algorithm, LL4, for curve fitting) to calculate $\delta$ (delta) scores/synergy scores for the interaction between Atovaquone and Idarubicin. The reference point in the ZIP model is zero (i.e. $\delta=0$ ), which implies zero interaction between the two drugs. Synergy scores lower than zero (i.e. $\delta<0$ ) imply a likely antagonistic interaction $(\delta<-10$ is considered most likely antagonistic), whilst $\delta>0$ shows additivity or synergy between the two tested drugs ( $\delta>10$ would be interpreted as most likely synergistic) (32).

\section{Statistical Analysis}

Sequencing quality control, mapping, quantification, and differential gene expression analysis were also performed by Novogene using HISAT2 software, RPKM calculation for each gene, DESeq2 and EdgeR package in $\mathrm{R}$ to generate lists with differentially expressed genes between Atovaquone treatment and control samples. Adjusted $p$-value $(\leq 0.05)$ and fold change $(\mathrm{FC})$ of $\geq 1.3$ and $\leq-1.3$ were used as thresholds to create the gene lists necessary for downstream analysis. Gene Ontology (GO) analysis was carried out by BinGO (33) in Cytoscape Software (34) where statistically significantly enriched nodes $(p-\leq 0.05)$ have been colour-coded (yellow for $5 \times 10^{-2}$ to dark orange for $5 \times 10^{-7}$ ). Gene set enrichment analysis (GSEA) was performed from generated lists with differentially expressed genes as described above using the GSEA software by the Broad Institute (35) (standard settings of 1,000 permutations, permutation type-gene_set, and Molecular Signature Database gene sets-H: Hallmark, C2: Curated gene sets, C5: ontology gene sets, C6: oncogenic signatures gene sets). The default FDR cut-off is 0.25 ; however, only gene sets with FDR $\leq 0.05$ were plotted. GraphPad Prism (v. 8) was used for figure preparation 
and statistical analysis (parametric unpaired Student's $t$-test for Seahorse and MTT data and paired Student's $t$-test for patient samples' standard cut-offs of $p$ - $\leq 0.05$ ).

\section{RESULTS}

\section{Atovaquone Demonstrates Anti- Leukaemic Properties In Vitro Through Inhibition of Mitochondrial Respiration}

The anti-proliferative effect of Atovaquone on REH cells was investigated first. We found that at $\mathrm{IC}_{50}$ concentration (Supplementary Figure 1A) Atovaquone exerts its anti-leukaemic effect through both deceleration of the cell cycle $(53.4 \pm 7.7 \%$ in G1phase in control cells $v s 67.6 \pm 3.5 \%$ in Ato-treated cells, $p=0.044$, as well as $29.6 \pm 6.3 \%$ of control cells in S-phase $v s 19.3 \pm 1.7 \%$ in Ato-treated cells, $p=0.05$, Supplementary Figure 1B). Ato also increased the number of apoptotic cells $(19.8 \pm 2.9 \%$ apoptotic cells in control $v s 30.2 \pm 6.6 \%$ in Ato-treated cells, $p=0.005$, Supplementary Figure 1C). In order to elucidate the potential mechanism of action of Ato. we examined the mitochondrial function of the cells following treatment with the drug for three days (Figure 1A). We found that Ato treatment led to a $\sim$ two-fold reduction of basal respiration (from $407.6 \pm 48.8$ to $219.4 \pm 53.9, p<$ 0.0001 ) and $\sim 3.7$-fold decrease in maximal respiration (from $813.8 \pm 93.1$ to $220.2 \pm 116, p<0.0001)$. It nearly completely abolished spare respiratory capacity (from $406.2 \pm 52.1$ to $0.8 \pm 63.6$, $p<0.0001$ ) and decreased ATP production by more than half (from $343 \pm 32.3$ to $137.6 \pm 79.6, p=0.0002$ ) (Figure $1 \mathrm{~B}$ and Supplementary Table 1 ).

RNA-seq (Figures 1C, D, Supplementary Figures 2 and 3, and Supplementary Table 2) aiming to elucidate the effect of Ato on REH cells showed that this drug changed the expression of nearly 2,000 genes compared to untreated cells (with cut-offs of adjusted $p$ value $<0.05$, FC $>1.3$ ). Gene ontology (GO) analysis revealed enrichment for cell metabolic processes (Figure 1C), apoptosis, cell cycle arrest, and a small cluster of oxidative stress genes (Supplementary Figures 2A-C), which is another confirmation of the phenotypic effects we observed. Further Gene Set Enrichment Analysis (GSEA) demonstrated-upregulation of the p53 pathway, downregulation of E2F, and G2M checkpoint (both related to cell cycle control), and of hypoxia gene sets (Figure 1D). We further found that Ato treatment may enhance fatty acid metabolism and, interestingly, downregulate RNA metabolic processes (Supplementary Figure 3).

Another B-cell precursor cALL cell line-Sup-B15 was analysed as well. We also made use of a Glucocorticoid-resistant sub-clone of Sup-B15 referred to as "Sup-PR" that was established in our laboratory (Methods and Supplementary Figure 1D). We observed that Ato had similar $\mathrm{IC}_{50}$ values as in $\mathrm{REH}$ cells $(\sim 30$ $\mu \mathrm{M}$, Supplementary Figure 1E) and elicited very similar changes in mitochondrial respiration and ATP production in both Sup-B15 and Sup-PR cells (Figures 2A, B). Of note, GC-resistance of around 1,000 times did not change the response to Ato, and we found significant reduction of basal and maximal respiration, as well as of ATP production in Sup-PR cells (Supplementary Table 3 and Figure 2B).

\section{Atovaquone Enhances the Effect of Standard Chemotherapeutics and Resensitises Resistant Cells to Glucocorticoids}

We also investigated if Ato may enhance the action of standard chemotherapeutics known to affect different aspects of cell metabolism. Thereby, we examined combinations with several chemotherapeutics which are part of the standard treatment regimen such as Methotrexate (targeting folic acid metabolism), Idarubicin (topoisomerase II inhibitor and inhibitor of complex I of the ETC), and Prednisolone (targeting glycolysis). We found marked synergistic effect (ZIP synergy score of 24.4) in REH cells only when Ato was in combination with Idarubicin (Figure 3A and Supplementary Figure $1 \mathrm{~F}$ for $\mathrm{IC}_{50}$ values) and not in any of the other two combinations (data not shown). Furthermore, this synergy had a marked effect on cell proliferation as well (from $1.96 \pm 0.4 \times 10^{5}$ live control cells on day 3 to $0.83 \pm 0.19 \times 10^{5}$ live cells treated with Ato and Idarubicin, p $<0.0001$, Figure $3 \mathbf{B}$ and Supplementary Table 4). We also investigated if this combination would work in Sup-B15 and Sup-PR cells (Supplementary Figure $1 F$ for $\mathrm{IC}_{50}$ values). Interestingly, whilst the two drugs demonstrated only additive effect on Sup-B15 cells (Figure 3C, synergy score of 3.6), Sup-PR proved more sensitive to this double treatment with a synergy score of 16.7 (Figure 3C).

Glucocorticoid (GC) resistance is believed to be one of the major causes of relapse in the clinic. Since we found that Sup-PR cells remain sensitive to Ato, we then investigated if Ato can resensitise these cells to Prednisolone. Therefore, we examined Ato-Pred double combination in Sup-B15 and Sup-PR cells. What we discovered was that whilst this combination in the parental Sup-B15 cells may even prove to be counterproductive (antagonistic ZIP score of -14.2-Figure 3D), this treatment had an additive effect on Sup-PR cells (score of 7-Figure 3D).

\section{Validation of the Effect of Atovaquone in Patient Samples}

Lastly, we attempted to validate our findings in a small number of patient samples available from the Oncohaematology Unit at the University Clinic of Paediatrics (Figures 4A-D and Supplementary Table 5). Importantly, we found that Ato is capable of reducing growth in primary lymphoblasts at $30 \mu \mathrm{M}$ concentrations (Figure 4A), which are lower than the maximum achievable plasma levels in vivo (see Discussion). Furthermore, we validated that Ato is capable of targeting mitochondrial respiration and ATP production in patient samples (Figures 4B, C and Supplementary Table 6). Lastly, we investigated the effect of combining Ato and Idarubicin in these samples. Interestingly, we found that this combination elicits a more variable response between the samples ranging from antagonistic (in one sample) to additive (in two samples), and synergistic (in one sample) (Figure 4D).

\section{DISCUSSION}

In summary, we have investigated the anti-proliferative activity of Atovaquone on two established B-cell precursor cALL cell 
A

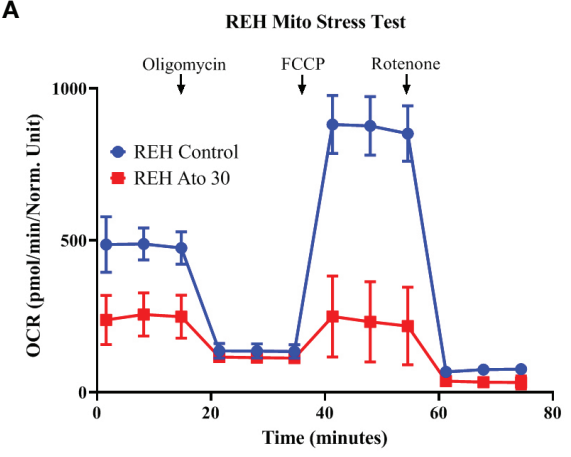

B

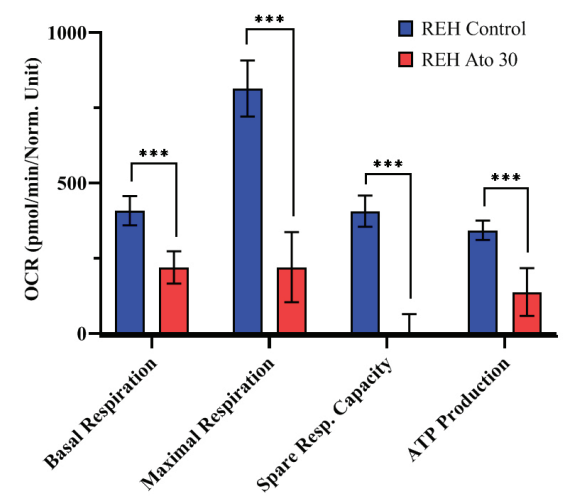

D

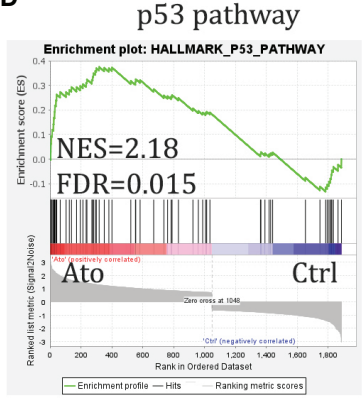

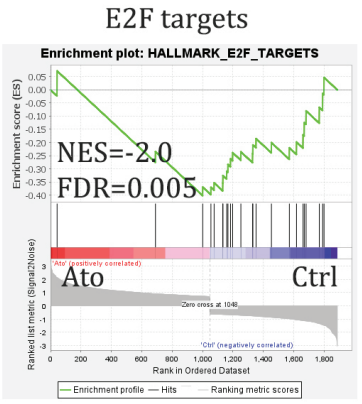

C

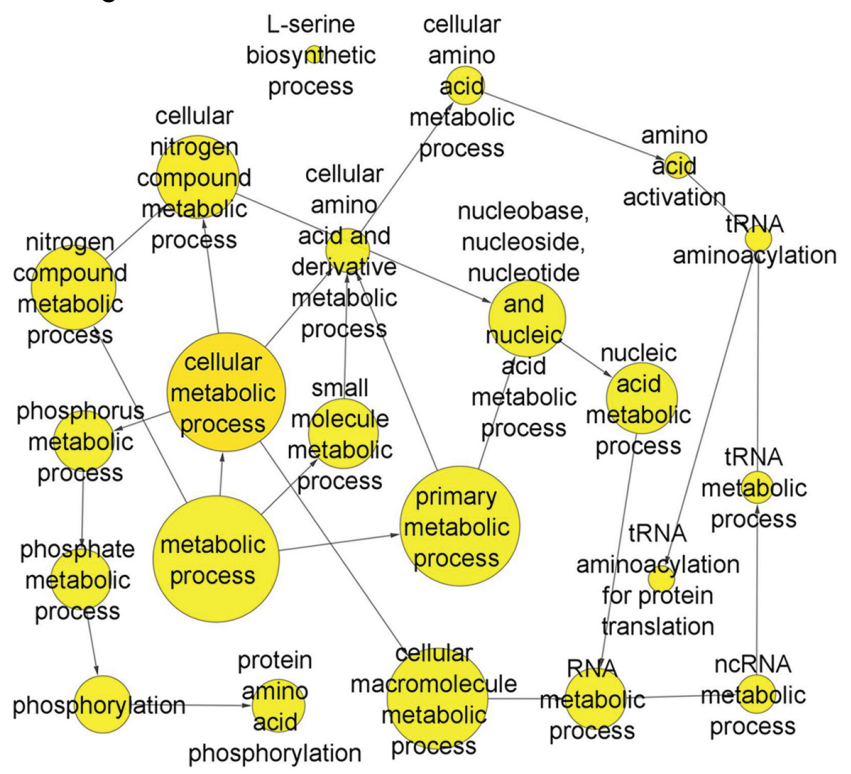

$5 \mathrm{E}-2$

$<5 \mathrm{E}-7$
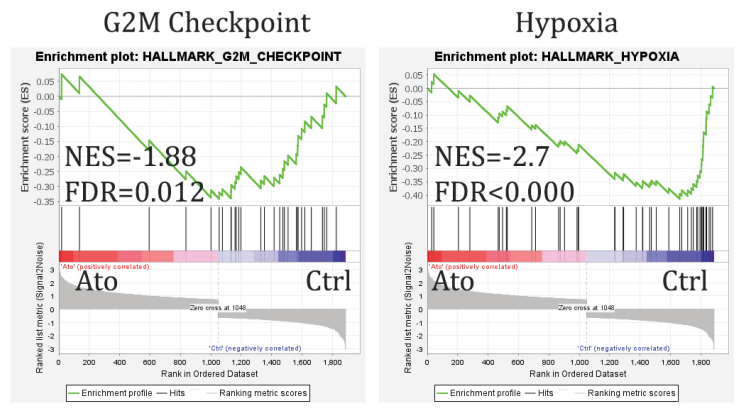

FIGURE 1 | Atovaquone treatment targets cell mithochondrial respiration and activates apoptosis and cell cycle arrest in REH cells. REH cells were treated with IC 50 concentrations of Atovaquone $(30 \mu \mathrm{M})$ for 3 days and compared to untreated control cells. (A) Their mitochondrial function was analysed with Seahorse XFp Analyser Instrument using the Mito Stress Test (Agilent, USA). (B) The results of the test showed reduction in basal and maximal respiration, spare respiratory capacity and ATP production after treatment with Ato. (C) Control and Ato-treated REH cells were collected on day 3 and RNA-sequencing was carried out. Gene ontology (GO) analysis with BinGO in Cytoscape showed a cluster of statistically significantly represented nodes related to metabolic processes as shown (statistically not significant nodes were removed from the cluster). Scale bar (yellow-orange) depicts $p$-values as annotated. (D) Gene set enrichment analysis (GSEA) was also performed with the GSEA software by the Broad Institute. The analysis demonstrates strong statistically significant (FDR q-value $<0.05)$ enrichment for the shown gene sets. Error bars represent mean with SD from biological triplicates in technical duplicates. ${ }^{\star \star \star} p<0.0005$, Student's $t$-test. NES, normalised enrichment score; FDR, false discovery rate q-value.

lines-REH and Sup-B15, as well as on a Prednisolone-resistant sub-clone of Sup-B15. We found that Ato elicited a very similar effect on mitochondrial respiration and ATP production in all three cell lines at concentrations of $\sim 30 \mu \mathrm{M}$. These findings are in line with previous in vitro studies $[10 \mu \mathrm{M}$ in breast cancer (26) and cervical cancer cells (36), $30 \mu \mathrm{M}$ in colorectal, pharyngeal and lung cancer cell lines $(27,37), \sim 15-50 \mu \mathrm{M}$ for glioblastoma cells (38), $20 \mu \mathrm{M}$ in retinoblastoma (28) and thyroid cancer cells (39)]. In particular, Ato reduced the levels of basal and maximal respiration, decreased the respiratory capacity of the cells and ATP production as measured by the Seahorse Mito Stress test. Even if we could carry out these experiments on a limited number of patient samples, we observed that Ato can induce similar changes of mitochondrial respiration in primary lymphoblasts as well.

GSEA and GO analyses in REH cells confirmed some of the phenotypic observations in our study-alterations in cell metabolism, induction of apoptosis, and cell cycle arrest 


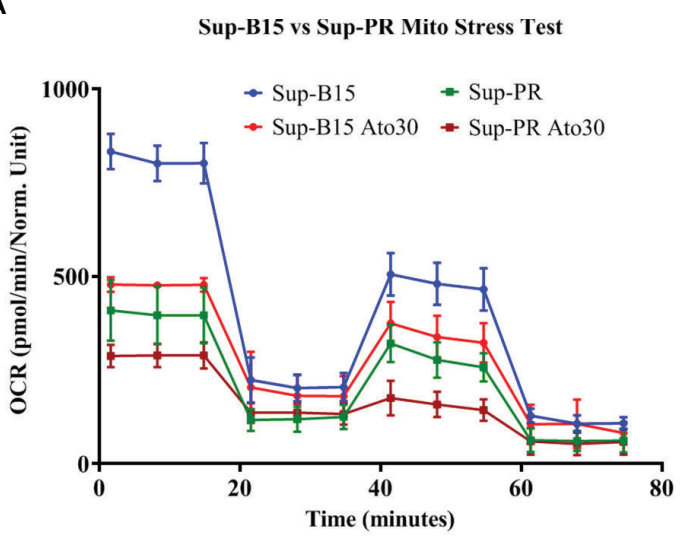

B

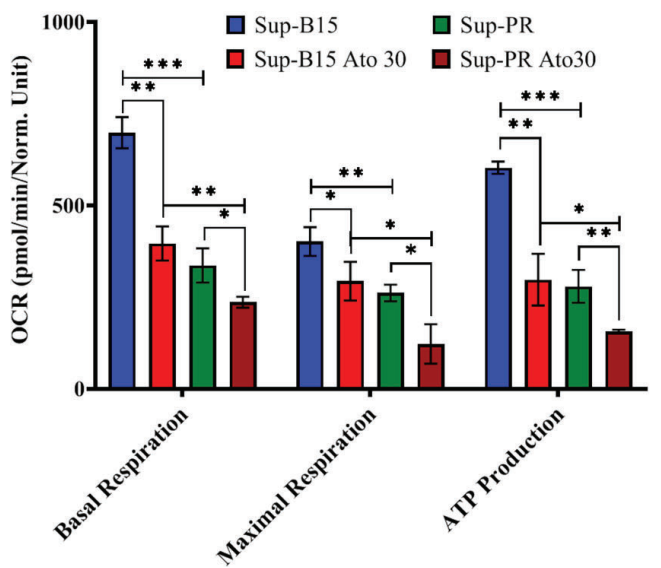

FIGURE 2 | Atovaquone decreases mitochondrial respiration and ATP production in Sup-B15 cells and Prednisolone-resistant Sup-PR cells. SupB15 and Sup-PR cells in biological duplicates were treated with $30 \mu \mathrm{M}$ of Ato for 3 days and compared to control untreated cells. (A) Sup-B15 cells and Sup-PR cells were assayed with Mito Stress Test on Seahorse XFp Analyser (Agilent, USA) and (B) were analysed for changes in mitochondrial respiration and ATP production. Error bars represent mean with SD from biological duplicates in technical duplicates. ${ }^{\star} p \leq 0.05,{ }^{\star \star} p \leq 0.005,{ }^{* \star} p<0.0005$, Student's t-test.

(Figure 1 and Supplementary Figure 2), and pointed to other interesting concomitant changes that may be due to Ato. Several papers from the past 5 years have investigated the anti-cancer effect of Ato. Most of them have focussed on inhibition of OxPhos, which we confirm herein as well, whilst two publications show that Ato may work through targeting the AKT-mTOR pathway (28). The interplay between mTOR complex 1 (mTORC1) and mitochondrial function and cell metabolism is well-documented (40). Activation of the AKTmTOR pathway, which is altered in up to one third of children with T-ALL (41), may lead to considerable changes in cell metabolism and upregulation of glycolysis (4, 42). Investigating the potential effect of Ato on this pathway was beyond the scope of our study. Nevertheless, one of the most downregulated genes we found $(\log 2=-2.1$, adjusted $p$-value $=0.0018)$ is PTEN and GSEA from our RNA-seq showed significant and strong downregulation of mTORC1 genes (Supplementary Table 2 and Supplementary Figure 3). Of note, there are links between mTORC1 and the MYC oncoprotein, as well as between overexpression of MYC and shifts in cALL metabolism (9). Therefore, it is noteworthy that our GSEA also revealed downregulation of MYC targets in Atotreated cells (Supplementary Figure 3).

Ato has been shown to alleviate hypoxia and sensitise cancer cells to radiotherapy (27). Our GSEA analysis highlights alterations of hypoxia as one of the top hits and indeed we see downregulation of the direct HIF1- $\alpha$ target $V E G F \alpha$ after treatment (Supplementary Table 2). There is evidence that radiation therapy for high-risk T-ALL patients may reduce rates of relapse (43). Given that hypoxia is a major factor for the efficacy of radiation therapy, that Ato reduces hypoxia in preclinical models $(27,44)$ and in our gene set enrichment analysis (Figure 1), Ato treatment may be worthy of further investigation in this clinical context of B-cell cALL too.

The combination of Ato with Idarubicin is another interesting aspect of our study. There is an independent confirmation of the synergy between Ato and anthracyclins coming from work on thyroid cancer cells. Lv and colleagues found that Ato and Doxorubicin are an effective strategy working through ETC and consequent STAT3 inhibition (39). Of note, Ato and Idarubicin were most effective in REH cells and Sup-PR cells, both of which are proliferating faster than Sup-B15. Therefore, it may be interesting to test this combination on matched patient samples at diagnosis and relapse, but this is beyond the scope of our current work.

GC-resistance is believed to be the main cause of drug-failure and relapse in cALL (45). Therefore, it was interesting to find that Sup-PR cells remain sensitive to Ato and that there was an additive effect of Ato and Prednisolone in this GC-resistant line, but not in the parental cells. Investigating the molecular mechanisms of resistance (e.g. why Sup-PR cells have significantly lower oxygen consumption rates than Sup-B15 cells-Figure 2) and of resensitisation to Prednisolone by Ato was another important aspect which fell out of the scope of our study. However, the effectiveness of Ato alone and in combination with Idarubicin or Prednisolone in GC-resistant cells is worthy of further pre-clinical investigations with matched samples and may add another context to a potential clinical trial.

Importantly, there are several key points related to the patient samples' data and the potential clinical applicability of this study that need to be addressed. Due to the small number of available patient samples, which all belong to the most common type of cALL and its most frequent molecular subtype, one of the main limitations of our work is the lack of depth and representativity of different entities within this heterogeneous disease. Even though the experimental data with these four patient samples cannot be over-interpreted and directly translated to the clinic, we show as a proof-of-concept that Ato can hit its target in primary lymphoblasts as well. We observe significant decrease in mitochondrial respiration and ATP production (Figure $4 \mathrm{~B}$ and 
A

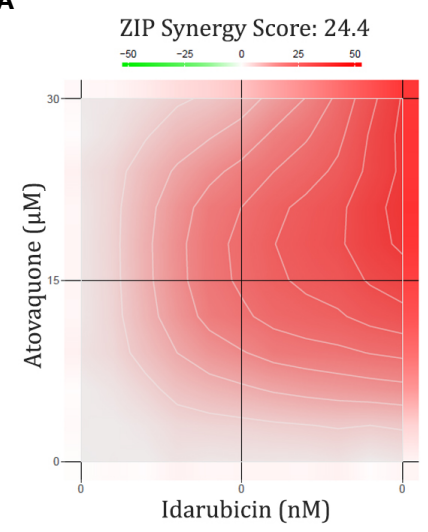

ZIP Synergy Score: 24.4

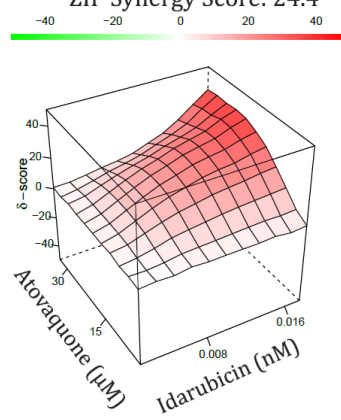

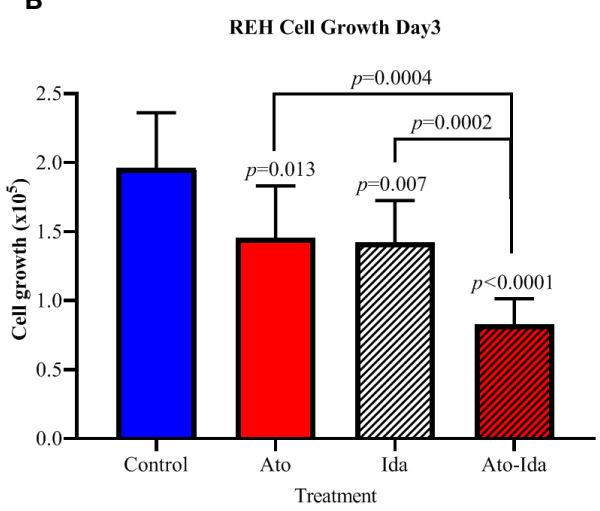

C
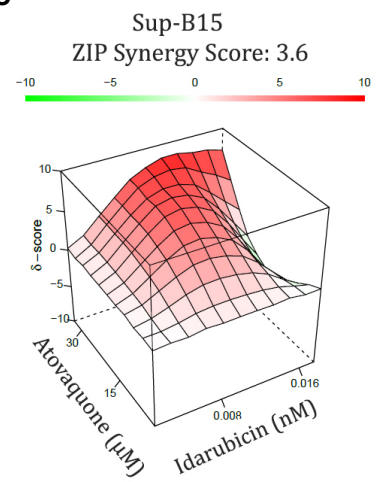

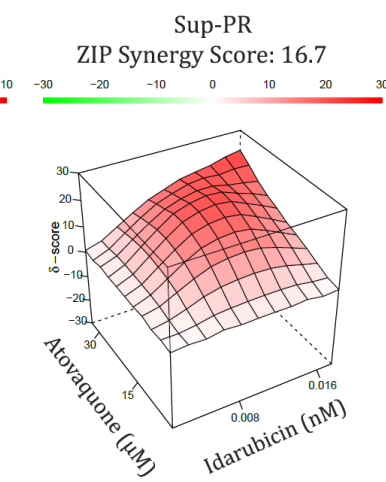

D

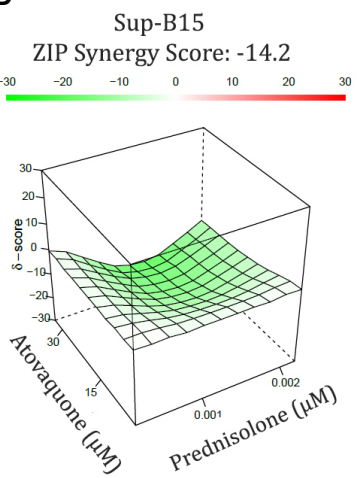

Sup-PR

ZIP Synergy Score: 7.0

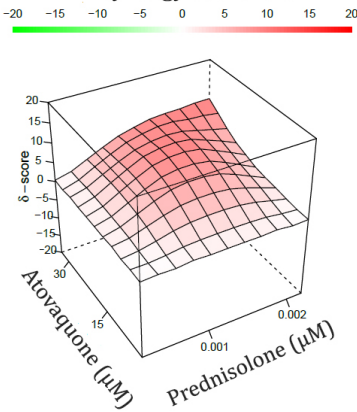

FIGURE 3 | Combination of Atovaquone with standard chemotherapeutics proves effective in REH Prednisolone resistant Sup-PR cells. (A) REH cells and (C) Sup$\mathrm{B} 15$ and Sup-PR cells were treated with combination of $\mathrm{IC}_{50}$ and $\mathrm{IC}_{25}$ concentrations of Atovaquone and Idarubicin in triplicates for three days and assessed by MTT test. The resulting cell viability matrix was tested by SynergyFinder. The data show \% inhibition based on percentage viable cells (left) and the calculated synergy score (right). (B) Cell growth of REH cells treated as shown was assessed by cell counting with Luna II Cell Counter (LogosBio, South Korea). Cell numbers were plotted as labelled in the figure. Error bars represent SD of the mean for $>6$ separate counts from triplicates. p-values were calculated with, Student's $t$-test. (D) Sup-B15 and Sup-PR cells were treated with $I_{50}$ and $I_{25}$ concentrations of Atovaquone and Prednisolone as single drugs and in combinations. Triplicates were analysed on day 3 by MTT and synergy scores were calculated by SynergyFinder as described above.

Supplementary Table 6), similarly to what we have in REH, SupB15, and Sup-PR cells. Nevertheless, the effect of Ato on cell viability in the patient samples (Figure $\mathbf{4 A}$ ) is not as prominent as in the cell lines. This modest decrease in cell proliferation, however, may be due to either sub-optimal concentrations of Ato or to the very slow cell division of cultured primary cells, which would not allow for the detection of potential cell cycle arrest (as seen in the fast proliferating $\mathrm{REH}$ cells) and would also account for the low OCR readings especially after Ato treatment. We also found that the combination of Ato and Idarubicin in patient samples elicits a range of responses-from antagonistic to synergistic. Besides potential differences in proliferation rates ex vivo, which may account for the lack of uniformity in the response to Ato and Idarubicin, the small number of patient samples does not allow for any speculations other than that there may be unidentified molecular and/or cytogenetic factors which may determine either advantageous or adverse results of the double treatments. Therefore, a larger cohort of patients including relapsed samples may be worth testing.
Lastly, the $\mathrm{IC}_{50}$ concentration we found for our cell lines of 30 $\mu \mathrm{M}(\sim 11 \mathrm{ug} / \mathrm{ml})$ (Supplementary Figures 1A, E) and the concentrations of maximum $90 \mu \mathrm{M}(\sim 33 \mathrm{ug} / \mathrm{ml})$ that we managed to test on some of the patient samples are within the clinically recorded levels for Ato. The steady-state plasma concentration in children achieved by daily administration of $30 \mathrm{mg} / \mathrm{kg}$ for 12 days is $37.1 \pm 10.9 \mathrm{ug} / \mathrm{ml}$ (as described in the prescribing information for Mepron ${ }^{\circledR}$ ). Other literature data suggests similar values-Cmax and Cmin of respectively $40.5 \pm 12.2$ and $34.0 \pm 11.7 \mathrm{ug} / \mathrm{ml}$ (of $30 \mathrm{mg} / \mathrm{kg}$ daily) (46). Furthermore, there is an interesting finding coming from a retrospective study of over 200 patients with AML who had undergone bone marrow transplantation. What Xiang et al. found was that administration of Ato for nearly two months as a part of standard anti-infection prophylaxis led to significant reduction of relapse rates ( $13 v s 23 \%, p=0.039$ ) (30). Therefore, considering the number of robust pre-clinical studies in other cancers, the good safety data for Ato and the clear effect on proliferation due to ETC inhibition, it is tempting to speculate 
A

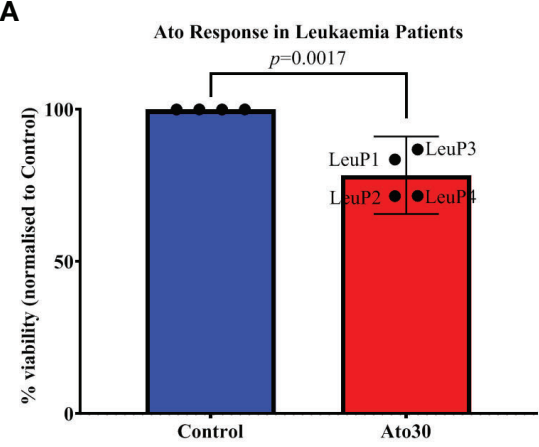

B

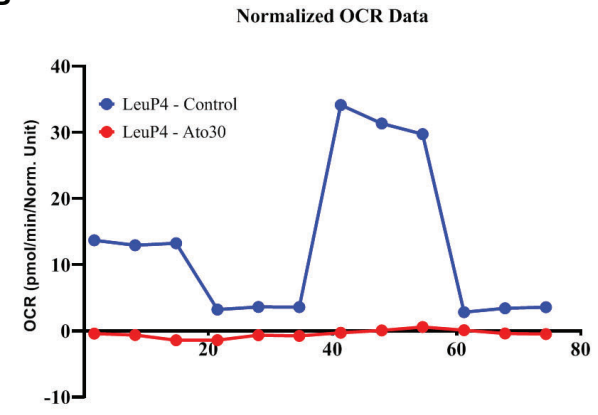

C Basal Respiration
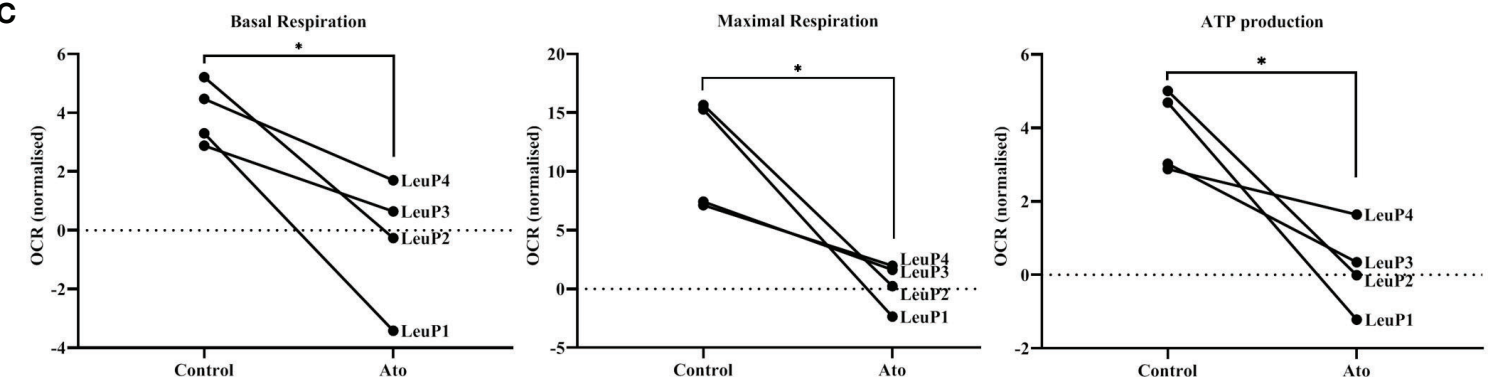

D

LeuP1

LeuP2

LeuP3

LeuP4

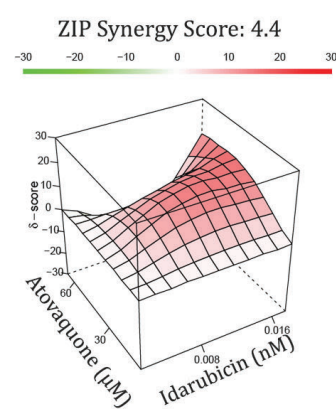
ZIP Synergy Score: -7.3

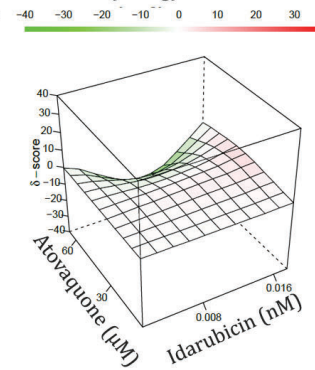

ZIP Synergy Score: 6.7

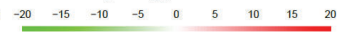

ZIP Synergy Score: 15.8
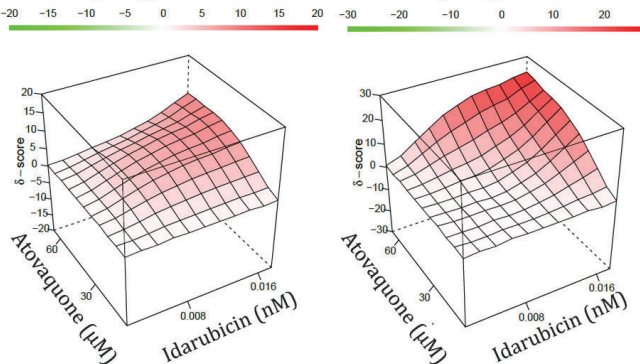

FIGURE 4 | Atovaquone shows efficacy in primary CALL lymphoblasts. (A) Isolated patient cells were cultured for 3 days with $30 \mu \mathrm{M}$ Atovaquone, and cell viability was assessed by MTT assay. Percentage live cells normalised to control (100\% viable) were calculated for each sample and plotted as shown. (B) A representative Mito Stress Test graph from patient \#2 showing the effect of $30 \mu \mathrm{M}$ Atovaquone on mitochondrial respiration. (C) Analysis of Mito Stress Tests for basal and maximal respiration as well as for ATP production as shown. The data from the highest tested concentration in each patient sample (60 $\mu \mathrm{M}$ for LeuP1 and $90 \mu \mathrm{M}$ for LeuP2, 3 and 4) was analysed. ${ }^{*} p \leq 0.05$, paired Student's $t$-test. (D) SynergyFinder analysis of the combinatorial effect of Atovaquone $(30$ and $60 \mu \mathrm{M})$ and Idarubicin $(0.0008$ and $0.0016 \mu \mathrm{M})$ in four patient samples as annotated. LeuP, Leukaemia patient.

that Ato would be worthy of further pre-clinical in vivo investigation and perhaps clinical trials as the ongoing one on Ato and radiation therapy (NCT02628080).

In conclusion, we present novel data demonstrating the antileukaemic effect of Atovaquone, the mechanism of action of the drug and the concomitant gene expression changes that may underpin the phenotypic changes observed. Furthermore, our results suggest that this FDA-approved ETC inhibitor may have additive or synergistic effect together with Idarubicin. GCresistant cells may be re-sensitised to Prednisolone when treated with Atovaquone. Therefore, the findings of this study may present a promising new therapeutic approach targeting cell metabolism, which may be worthy of further investigation.

\section{DATA AVAILABILITY STATEMENT}

The original contributions presented in the study are publicly available. This data can be found here: https://www.ncbi.nlm.nih. gov/geo/query/acc.cgi?acc=GSE161959.

\section{ETHICS STATEMENT}

The studies involving human participants were reviewed and approved by the Ethics committee at Medical University of Plovdiv. Written informed consent to participate in this study was provided by the participants' legal guardian/next of kin. 


\section{AUTHOR CONTRIBUTIONS}

YS, TI, TS, KP, and VS designed the study. YS, TI, HB, and KG developed the methodology. YS, TI, TS, KP, and VS analysed the data. YS, VS, TS, and KP wrote and edited the manuscript. All authors contributed to the article and approved the submitted version.

\section{FUNDING}

This project was funded by the Bulgarian National Science Fund (project No. KP-06-M21/9/19.12.18), and supported by the National University Complex for Biomedical and Applied Research with participation in BBMRI-ERIC, NUCBPI-BBMRI.BG, (contracts No. D01-285/ 17.12.2019 and

\section{REFERENCES}

1. Warburg O, Wind F, Negelein E. The Metabolism of Tumors in the Body. J Gen Physiol (1927) 8:519-30. doi: 10.1085/jgp.8.6.519

2. DeBerardinis RJ, Chandel NS. Fundamentals of cancer metabolism. Sci $A d v$ (2016) 2:e1600200. doi: 10.1126/sciadv. 1600200

3. Luengo A, Gui DY, Vander Heiden MG. Targeting Metabolism for Cancer Therapy. Cell Chem Biol (2017) 24:1161-80. doi: 10.1016/j.chembiol.2017.08.028

4. Sbirkov Y, Burnusuzov H, Sarafian V. Metabolic reprogramming in childhood acute lymphoblastic leukemia. Pediatr Blood Cancer (2020) 67:e28255. doi: $10.1002 / \mathrm{pbc} .28255$

5. Gu Z, Churchman ML, Roberts KG, Moore I, Zhou X, Nakitandwe J, et al. PAX5-driven subtypes of B-progenitor acute lymphoblastic leukemia. Nat Genet (2019) 51:296-307. doi: 10.1038/s41588-018-0315-5

6. Boag JM, Beesley AH, Firth MJ, Freitas JR, Ford J, Hoffmann K, et al. Altered glucose metabolism in childhood pre-B acute lymphoblastic leukaemia. Leukemia (2006) 20:1731-7. doi: 10.1038/sj.leu.2404365

7. Kodron A, Ghanim M, Krawczyk KK, Stelmaszczyk-Emmel A, Tonska K, Demkow U, et al. Mitochondrial DNA in pediatric leukemia patients. Acta Biochim Pol (2017) 64:183-7. doi: 10.18388/abp.2016_1444

8. Jenkinson S, Koo K, Mansour MR, Goulden N, Vora A, Mitchell C, et al. Impact of NOTCH1/FBXW7 mutations on outcome in pediatric T-cell acute lymphoblastic leukemia patients treated on the MRC UKALL 2003 trial. Leukemia (2013) 27:41-7. doi: 10.1038/leu.2012.176

9. Herranz D, Ambesi-Impiombato A, Sudderth J, Sanchez-Martin M, Belver L, Tosello V, et al. Metabolic reprogramming induces resistance to antiNOTCH1 therapies in T cell acute lymphoblastic leukemia. Nat Med (2015) 21:1182-9. doi: 10.1038/nm.3955

10. Dyczynski M, Vesterlund M, Bjorklund AC, Zachariadis V, Janssen J, GallartAyala $\mathrm{H}$, et al. Metabolic reprogramming of acute lymphoblastic leukemia cells in response to glucocorticoid treatment. Cell Death Dis (2018) 9:846. doi: 10.1038/s41419-018-0625-7

11. Aoki S, Morita M, Hirao T, Yamaguchi M, Shiratori R, Kikuya M, et al. Shift in energy metabolism caused by glucocorticoids enhances the effect of cytotoxic anti-cancer drugs against acute lymphoblastic leukemia cells. Oncotarget (2017) 8:94271-85. doi: 10.18632/oncotarget.21689

12. Yadav N, Kumar S, Marlowe T, Chaudhary AK, Kumar R, Wang J, et al. Oxidative phosphorylation-dependent regulation of cancer cell apoptosis in response to anticancer agents. Cell Death Dis (2015) 6:e1969. doi: 10.1038/ cddis.2015.305

13. Panosyan EH, Grigoryan RS, Avramis IA, Seibel NL, Gaynon PS, Siegel SE, et al. Deamination of glutamine is a prerequisite for optimal asparagine deamination by asparaginases in vivo (CCG-1961). Anticancer Res (2004) 24:1121-5.

14. Hermanova I, Arruabarrena-Aristorena A, Valis K, Nuskova H, AlberichJorda M, Fiser K, et al. Pharmacological inhibition of fatty-acid oxidation
DO1-395/ 18.12.2020) within the National Road Map For Research Infrastructure.

\section{ACKNOWLEDGMENTS}

We would like to thank St George University Hospital (Plovdiv) for providing patient samples and clinical data and Novogene UK for RNA-sequencing and initial data analysis.

\section{SUPPLEMENTARY MATERIAL}

The Supplementary Material for this article can be found online at: https://www.frontiersin.org/articles/10.3389/fonc.2021. 632181/full\#supplementary-material synergistically enhances the effect of 1-asparaginase in childhood ALL cells. Leukemia (2016) 30:209-18. doi: 10.1038/leu.2015.213

15. Umerez M, Gutierrez-Camino A, Munoz-Maldonado C, Martin-Guerrero I, Garcia-Orad A. MTHFR polymorphisms in childhood acute lymphoblastic leukemia: influence on methotrexate therapy. Pharmgenomics Pers Med (2017) 10:69-78. doi: 10.2147/PGPM.S107047

16. Kato M, Manabe A. Treatment and biology of pediatric acute lymphoblastic leukemia. Pediatr Int (2018) 60:4-12. doi: 10.1111/ped.13457

17. Bhojwani D, Pui CH. Relapsed childhood acute lymphoblastic leukaemia. Lancet Oncol (2013) 14:e205-217. doi: 10.1016/S1470-2045(12)70580-6

18. Ashburn TT, Thor KB. Drug repositioning: identifying and developing new uses for existing drugs. Nat Rev Drug Discov (2004) 3:673-83. doi: 10.1038/ nrd1468

19. Pushpakom S, Iorio F, Eyers PA, Escott KJ, Hopper S, Wells A, et al. Drug repurposing: progress, challenges and recommendations. Nat Rev Drug Discov (2019) 18:41-58. doi: 10.1038/nrd.2018.168

20. Nowak-Sliwinska P, Scapozza L, Ruiz i Altaba A. Drug repurposing in oncology: Compounds, pathways, phenotypes and computational approaches for colorectal cancer. Biochim Biophys Acta Rev Cancer (2019) 1871:434-54. doi: 10.1016/j.bbcan.2019.04.005

21. Rudrapal M, Khairnar SJ, Jadhav AG. Drug Repurposing (DR): An Emerging Approach in Drug Discovery. IntechOpen (2020) 1-20. doi: 10.5772/ intechopen. 93193

22. Degos L, Wang ZY. All trans retinoic acid in acute promyelocytic leukemia. Oncogene (2001) 20:7140-5. doi: 10.1038/sj.onc.1204763

23. Schenk T, Chen WC, Gollner S, Howell L, Jin L, Hebestreit K, et al. Inhibition of the LSD1 (KDM1A) demethylase reactivates the all-trans-retinoic acid differentiation pathway in acute myeloid leukemia. Nat Med (2012) 18:605-11. doi: 10.1038/nm.2661

24. Haile LG, Flaherty JF. Atovaquone: a review. Ann Pharmacother (1993) 27:1488-94. doi: 10.1177/106002809302701215

25. Nixon GL, Moss DM, Shone AE, Lalloo DG, Fisher N, O'Neill PM, et al. Antimalarial pharmacology and therapeutics of atovaquone. J Antimicrob Chemother (2013) 68:977-85. doi: 10.1093/jac/dks504

26. Fiorillo M, Lamb R, Tanowitz HB, Mutti L, Krstic-Demonacos M, Cappello $\mathrm{AR}$, et al. Repurposing atovaquone: targeting mitochondrial complex III and OXPHOS to eradicate cancer stem cells. Oncotarget (2016) 7:34084-99. doi: 10.18632/oncotarget.9122

27. Ashton TM, Fokas E, Kunz-Schughart LA, Folkes LK, Anbalagan S, Huether $\mathrm{M}$, et al. The anti-malarial atovaquone increases radiosensitivity by alleviating tumour hypoxia. Nat Commun (2016) 7:12308. doi: 10.1038/ncomms12308

28. Ke F, Yu J, Chen W, Si X, Li X, Yang F, et al. The anti-malarial atovaquone selectively increases chemosensitivity in retinoblastoma via mitochondrial dysfunction-dependent oxidative damage and Akt/AMPK/mTOR inhibition. Biochem Biophys Res Commun (2018) 504:374-9. doi: 10.1016/j.bbrc. 2018.06.049 
29. Das S, Dielschneider R, Chanas-LaRue A, Johnston JB, Gibson SB. Antimalarial drugs trigger lysosome-mediated cell death in chronic lymphocytic leukemia (CLL) cells. Leuk Res (2018) 70:79-86. doi: 10.1016/ j.leukres.2018.06.005

30. Xiang M, Kim H, Ho VT, Walker SR, Bar-Natan M, Anahtar M, et al. Gene expression-based discovery of atovaquone as a STAT3 inhibitor and anticancer agent. Blood (2016) 128:1845-53. doi: 10.1182/blood-2015-07660506

31. Ianevski A, Giri AK, Aittokallio T. SynergyFinder 2.0: visual analytics of multi-drug combination synergies. Nucleic Acids Res (2020) 48:W488-93. doi: 10.1093/nar/gkaa216

32. Yadav B, Wennerberg K, Aittokallio T, Tang J. Searching for Drug Synergy in Complex Dose-Response Landscapes Using an Interaction Potency Model. Comput Struct Biotechnol J (2015) 13:504-13. doi: 10.1016/j.csbj.2015.09.001

33. Maere S, Heymans K, Kuiper M. BiNGO: a Cytoscape plugin to assess overrepresentation of gene ontology categories in biological networks. Bioinformatics (2005) 21:3448-9. doi: 10.1093/bioinformatics/bti551

34. Shannon P, Markiel A, Ozier O, Baliga NS, Wang JT, Ramage D, et al. Cytoscape: a software environment for integrated models of biomolecular interaction networks. Genome Res (2003) 13:2498-504. doi: 10.1101/ gr. 1239303

35. Subramanian A, Tamayo P, Mootha VK, Mukherjee S, Ebert BL, Gillette MA, et al. Gene set enrichment analysis: a knowledge-based approach for interpreting genome-wide expression profiles. Proc Natl Acad Sci U S A (2005) 102:15545-50. doi: 10.1073/pnas.0506580102

36. Tian S, Chen H, Tan W. Targeting mitochondrial respiration as a therapeutic strategy for cervical cancer. Biochem Biophys Res Commun (2018) 499:101924. doi: 10.1016/j.bbrc.2018.04.042

37. Coates JTT, Rodriguez-Berriguete G, Puliyadi R, Ashton T, Prevo R, Wing A, et al. The anti-malarial drug atovaquone potentiates platinum-mediated cancer cell death by increasing oxidative stress. Cell Death Discov (2020) 6:110. doi: 10.1038/s41420-020-00343-6

38. Takabe H, Warnken ZN, Zhang Y, Davis DA, Smyth HDC, Kuhn JG, et al. A Repurposed Drug for Brain Cancer: Enhanced Atovaquone Amorphous Solid Dispersion by Combining a Spontaneously Emulsifying Component with a Polymer Carrier. Pharmaceutics (2018) 10(2):60. doi: 10.3390/ pharmaceutics 10020060

39. Lv Z, Yan X, Lu L, Su C, He Y. Atovaquone enhances doxorubicin's efficacy via inhibiting mitochondrial respiration and STAT3 in aggressive thyroid cancer. J Bioenerg Biomembr (2018) 50:263-70. doi: 10.1007/s10863-0189755-y

40. de la Cruz Lopez KG, Toledo Guzman ME, Sanchez EO, Garcia Carranca A. mTORC1 as a Regulator of Mitochondrial Functions and a Therapeutic Target in Cancer. Front Oncol (2019) 9:1373. doi: 10.3389/fonc.2019.01373

41. Zuurbier L, Petricoin EF 3rd, Vuerhard MJ, Calvert V, Kooi C, BuijsGladdines JG, et al. The significance of PTEN and AKT aberrations in pediatric T-cell acute lymphoblastic leukemia. Haematologica (2012) 97:1405-13. doi: 10.3324/haematol.2011.059030

42. Elstrom RL, Bauer DE, Buzzai M, Karnauskas R, Harris MH, Plas DR, et al. Akt stimulates aerobic glycolysis in cancer cells. Cancer Res (2004) 64:3892-9. doi: 10.1158/0008-5472.CAN-03-2904

43. Kassick M, Koffer PP, Kinsella TJ. Trends in the Use of Radiation Therapy for Pediatric T-Cell Acute Lymphoblastic Leukemia and Impact on Survival: A Population-Based Analysis. Int J Radiat Oncol (2016) 96:E553-554. doi: 10.1016/j.ijrobp.2016.06.2014

44. Ashton TM, McKenna WG, Kunz-Schughart LA, Higgins GS. Oxidative Phosphorylation as an Emerging Target in Cancer Therapy. Clin Cancer Res (2018) 24:2482-90. doi: 10.1158/1078-0432.CCR-17-3070

45. Goto H. Childhood relapsed acute lymphoblastic leukemia: Biology and recent treatment progress. Pediatr Int (2015) 57:1059-66. doi: 10.1111/ ped.12837

46. Hughes W, Dorenbaum A, Yogev R, Beauchamp B, Xu J, McNamara J, et al. Phase I safety and pharmacokinetics study of micronized atovaquone in human immunodeficiency virus-infected infants and children. Pediatric AIDS Clinical Trials Group. Antimicrob Agents Chemother (1998) 42:1315-8. doi: 10.1128/AAC.42.6.1315

Conflict of Interest: The authors declare that the research was conducted in the absence of any commercial or financial relationships that could be construed as a potential conflict of interest.

Copyright (c) 2021 Sbirkov, Ivanova, Burnusuzov, Gercheva, Petrie, Schenk and Sarafian. This is an open-access article distributed under the terms of the Creative Commons Attribution License (CC BY). The use, distribution or reproduction in other forums is permitted, provided the original author(s) and the copyright owner(s) are credited and that the original publication in this journal is cited, in accordance with accepted academic practice. No use, distribution or reproduction is permitted which does not comply with these terms. 